\section{The Biochemical Model of Life Loses: Its Scientific Value}

\section{Abstract}

The human biological system creates the image structure of the world not only based on the electromagnetic and acoustic wave received by the senses, but also based on the soliton, spin and bio-plasm wave. This is a new face of knowledge for psychology and philosophy and can be directed to new research, hitherto unknown. The biochemical model is not sufficient to explain the perception and nature of mental processes, because it is too poor in scientific concepts and is based on classical science. The perception and nature of mental processes should be explained outside the biochemical model, also based on the bioelectronic model, and the IT and cybernetic paradigm.

Keywords: Bioelectronic processes; Bioplasma; Biocomputer; Perception; Soliton; Magnon

Received: January 11, 2019; Accepted: January 29, 2019; Published: February 01, 2019

\section{Adamski A* \\ Faculty of Ethnology and Educational Science, University of Silesia in Katowice, Cieszyn, Poland}

\author{
*Corresponding author: \\ Dr. Adam Adamski
}

” a_adamski@o2.pl

Faculty of Ethnology and Educational Science, University of Silesia in Katowice, Cieszyn, Poland.

Tel: 338546150

Citation: Adamski A (2019) The Biochemical Model of Life Loses: Its Scientific Value. Insights Biomed Vol.4 No.1:6

\title{
Introduction
}

\section{Defects in the biochemical model of life}

Better understanding of human mental life is possible thanks to the laws of quantum physics, which psychology must accept. In order to explain the mechanism of human brain's action and the nature of consciousness in terms of quantum physics and bioelectronic processes it is required to get rid of the baggage of traditional concepts and practices imposed by classical physics and psychology.

Biochemical model explains the intricate mechanisms of mental life. However, it still cannot explain the process of transition from inanimate to living matter. The answer to the following questions is still unknown. Where is the threshold and what is its essence? What role is played by biochemical processes in the unity of soma and consciousness and what is consciousness's impact on the soma, and vice versa? Other mental processes are also very difficult to explain since their nature cannot be explained using biochemical model of life and it is inexplicable on the basis of biochemical interactions. Definitely, it is easier to describe them in the light of quantum processes - including the wave physics $[1,2]$.

This model shows that the molecules (protein, melanin, DNA, RNA) that build cell structures and determine the molecular surface of chemical reactions, are also electronic material that has piezoelectric, pyroelectric, ferroelectric and semiconductor properties [3].
Biological systems have a network of molecular pyroelectrics, piezoelectrics and semiconductors which functions as an environment for the transformation of mechanical, thermal, electromagnetic, chemical energy in to electrical one. Every living organism has acquired piezoelectric, pyroelectric and semiconductor properties that are needed to produce bioelectronic processes that are necessary for the functioning of the body. These processes occur throughout the body, but are particularly noticeable in the following systems: cardiovascular, musculo-skeletal while walking, nervous - mechanoreption, baroreception, visual perception, sense of hearing, in the process of breathing, and during sexual arousal, etc.

Light and electric field are the most important factors in regulating the biosynthesis of melanin whereas the absence of these factors leads to the biosynthesis of melatonin. Melanin reduces the amount of free radicals in one's biological system. They fulfill an important role in locating information in the nucleus. Radical reactions modify the settings of spin nuclei and particles which in turn activate the spin field. Signal transmission does not have to take place solely under the influence of electro-magnetic and acoustic wave or electric field. There could also appear soliton waves, as a carrier of information, which would have an impact on the energy-informational system of an individual and its behavior [4-6].

Author's implications prove that a living organism can be perceived as a complex electronic device similar to technical 
devices, whereas biological materials (proteins, DNA, RNA) - as components of electronic devices. These arguments allow us to state that a biological system can be considered to be a quantum computer that functions on the basis of entangled quantum states and optoelectronic phenomena. Melanin and neuromelanin are involved in the central control of all biological, physiological and psychological processes. Numerous modular communication systems and signaling pathways that transmit signals into cells are generated under the influence of light. Melanin and neuromelanin function as multireceptors of a full range of electromagnetic, acoustic, and soliton waves, torsion fields and bioplasma, which does not receive so much information as the senses do but receive it constantly. The role of photoreceptors, receptors of hearing and touch is limited to a single reception of a stimulus, whereas melanin and neuromelanin play an integrative function, combining stimulus elements into a whole, namely combining movement with space and time, sound with light, space and time. From the psychological point of view, melanin and neuromelanin are responsible for the entire process of adaptation to the environment, mental development, the development of attention and perceptual experience, which, together with an increase in melanin and neuromelanin, acquire better sharpness and quality. Bioplasma controls these processes [7].

Biochemical model of life is largely based on the biochemical processes occurring in biological system. In addition to the role of electrons, it does not take into account, the role of photons and phonons. Therefore, the energy balance in the biological system has to be a place not only on chemical bonds and the electrons are flowing, but also the energy of photons, phonons, solitons and magnons. Biochemical model of life has already made some concessions to the electrochemical, but there is a need for the following concessions to the piezoelectricity pyroelectricity semi conductivity, superconductivity, liquid crystals, to explain the electrical and magnetic properties of biological matter, and a concession to the electromagnetic fields, spin, bioplasm, as well as for the acoustic wave, electromagnetic and solitons. Quantum psychology is able to accept the thesis that consciousness in its process of operation may behave as a wave or a particle, and it can also include linear and nonlinear processes. Bioplasm has the ability to detach from the body and create its own bioplasmoid containing bioholograms It is not very clear whether BoseEinstein condensate works with those altered states. Bioplasm is a factor in managing these processes, giving them the logic of action that are tailored to different states of consciousness and human behavior [8].

\section{Historical development of solitons}

Let's start with a brief historical overview. The first recorded solitary wave was observed in the 1834 when a young engineer named John Scott Russell was hired for a summer job to investigate how to improve the efficiency of designs for barges that were designated to ply canals-particularly the Union Canal near Edinburgh, Scotland $[9,10]$. One August day, the tow rope that was connecting the mules to the barge broke and the barge suddenly stopped-but the mass of water in front of its blunt prow rolled forward with great velocity, assuming the form of a large solitary elevation, a rounded, smooth and well defined heap of water, which continued its course along the channel without change of form or diminution of speed [11].

Russell pursued this serendipitous observation and followed it [the launched 'Wave of Translation'] on horseback, and overtook it still rolling on at a rate of some eight or nine miles per hour, preserving its original form some thirty feet long and a foot to a foot and a half in height. He then conducted controlled laboratory experiments using a wave tank and quantified the phenomenon in publication [11]. He demonstrated four facts:

1. The solitary waves that he observed had a hyperbolic secant shape.

2. A sufficiently large initial mass of water can produce two or more independent near-solitary waves that separate in time.

3. Solitary waves can cross each other "without change of any kind".

4. In a shallow water channel of height $h$, a solitary wave of amplitude A travels at a speed of $[g(A+h)] 1 / 2$, where $g$ is the gravitational acceleration. That is, larger-amplitude waves move faster than smaller ones-a nonlinear effect.

In 1895, Dutch physicist Diederick Korteweg and his student Gustav de Vries (KdV) derived a nonlinear partial differential equation (PDE), that now bears their name [10]. Korteweg and de Vries argued that the KdV equation (1) could describe Russell's experiments. Equation (1) shows that the rate of change of the wave's height in time is governed by the sum of two terms: a nonlinear one (the amplitude effect) and a dispersive one (the effect that causes waves of different wave-lengths to travel with different velocities). Korteweg and de Vries found a periodic solution in addition to a solitary-wave solution that resembled the wave that Russell had followed. These solutions arose as a result of a balance between nonlinearity and dispersion. Their work and Russell's observations fell into obscurity and were ignored by mathematicians, physicists, and engineers studying water waves until 1965 when Norman Zabusky and Martin Kruskal published their numerical solutions of the KdV equation (and invented the term "soliton" [12].

The effect of solitons was observed in various materials, of different dimensionality, shape, spiralling, colliding, fusing, splitting, in homogeneous media, periodic systems, and waveguides, in photorefractive crystals $[13,14]$.

Pang investigated-under high voltage-the influence of EMF over the collagen properties, solitons emerged in the infra-red spectrum of collagen $\left(480-2000 \mathrm{~cm}^{-1}\right)$. In this way, Pang Xiao Feng et al., were able to state that the influence of an externally applied electric field could justify solitons' bio-energetic transportation in collagen. This is done by means of the electrical properties amino-acids have from protein molecules [15].

From these studies it was concluded that the soliton induction of collagen can act as an optical fiber, causing other nonlinear effects as a waveguide mechanism acting over the fibers of collagen. Nowadays, there exist mathematical patterns that allow us to understand the propagation of non-linear stimulations and 
their influence over the transcription of DNA. As we saw before, during such process, about 15 to 20 pairs are open making up a "transcription bubble" that is propagated as a "solitary wave" as represented by the Sine-Gordon nonlinear equations and Nash Game Theory $[16,17]$.

In 1971, the Russian scientist A.S. Davydov proposed the soliton theory to explain the transport of "energy packages" in very long helical molecules, such as amino acids. According to this theory, bio-energy transports solitons along the helical protein molecules by the "stretching vibration" of amide residues affected by energy released from ATP hydrolysis, which causes a vibrational quantum called excitons while the deformation of amino acid residues also occur simultaneously [18].

The works Pougeta i Maugina contributed significantly to the development of the field of solitons $[19,20]$. In a transparent manner from the mathematical and physical side, the soliton is discussed. It is understood as a single moving wave that propagates with a small loss of energy unchanged, they are localized and require the presence of a physical environment as a carrier of information, therefore they cannot propagate in a vacuum, which is not required by other elementary particles. During the transmission, solitons transfer not elementary particles, but the information itself contained in the change of conformation, i.e., in the self-formation of the water, plasma, gas, light, etc. In infinity they seek to zero, or to a certain constant. They can strongly interact with other solitons, but after the collision they return to their original shape, that is they retain their shape and speed. Pouget and Maugina showed the effect of solitons in ferroelectrics, along with electroacoustic interaction, which is conditioned by piezoelectric and electro-curriction. They point to the domain structure of the medium, which determines the size and intensity of the soliton wave. The movement of solitons is affected by the density and thickness of the biological membrane in the cell, because it determines the size of the piezoelectric effect from which the electric field flows, interacting with the solitons. Using the Lax-Wendroff mathematical equations, they illustrate analytical considerations and collision model's solitonantysoliton, collision soliton-soliton, the action of a single solitona in various media [21].

Piezoelectricity, priroelectricity, ferroelectricity and semiconductivity are the constant properties of biological structures that determine the structure and function of the biological system and are responsible for different mental states of varying magnitude and intensity [2,22].

Solitons are generated in nonlinear optical centres and in Bose - Einstein concentrates. Strong laser waves, the degree of nonlinearity and high concentration of atoms in a Bose-Einstein condensate have an impact on the formation of multi-dimensional solitons. Currently, the greatest degree of non-linearity is achieved by organic substances in which electrons appear likely to travel long distances. Dimensional solitons owe their existence and permanence to a balance of two forces. Dispersion tends to expand, while non-linearity seeks to compress solitons. Such a soliton can be obtained, directing a laser beam at appropriately selected half of the condensate [23].
It was noted that the soliton could generate an electromagnetic wave, or absorb it, resulting in the formation of a continuous conduction medium and information transfer at on distance [24]

\section{Soliton and spin wave as an information carrier in perception}

Exposing melanin to light results in increased EPR signal. This is due to an increase in stationary concentration of radicals which are becoming spin generators, thus also emitting spin waves which take on the role of an information relay. Radical generation effectiveness increases significantly as wavelength decreases in the 230 to $600 \mathrm{~nm}$ range.

EPR spectroscopy is one of the most promising methods for detecting and characterizing melanin in biological material. It is a non-destructive method that features high sensitivity of free radical detection in a sample. The EPR method allows for determining the type, properties and concentration of paramagnetic centres in melanins, spin-spin interaction between free radicals and spin-network interaction of unpaired electrons with diamagnetic polymer molecules $[25,26]$.

Free radical concentration in melanin changes depending on environment $\mathrm{pH}$, biopolymer exposure to light, degree of hydration, temperature, metal ion content, therapeutic substances and presence of oxygen in the environment of a sample [27]. So free radical intensity increases as light intensifies, whereas decreases in temperature extends the life span of free radicals [28].

Spin movement generated in melanin during its synthesis, as well as from various reactive oxygen species, contributes to the generation of spin waves which are carriers for solitons. On the other hand, electromagnetic waves are carriers for phonons i.e., an acoustic wave. This phenomenon is used in television broadcasting which provides images and sounds. A soliton is not an independent entity, it needs a physical environment which is provided, among other things, by spin waves whose quantum is magnons [29-34].

\section{Discussion}

According to Popp, in DNA there is a biological laser which plays an important role in the functioning of bioplasma and the formation of the structure of consciousness. DNA uses different frequencies of electromagnetic waves and is a source of information for cells. In a healthy body the state of photon emission is more consistent than in a sick person. In respect of cancer cells, the intensity of biophoton emission is increased, and is not controlled by the biological system [30].

The author of this paper thinks that the laser in DNA generates solitons and coherent light and determines ways to manage solitons which are involved in the formation of conscious states and all mental processes. The high consistency of coherent light is well correlated with spin pumping to bioplasma and high capacity solitons management. Spin pumping creates a problem since the spin polarization is suitably unstable in time, so it is important to control the role of spin relaxation processes and creating act consciousness [31]. 


\section{Conclusion}

In modern science existing biosystems are considered at corpuscular structure level, with energy and information structures being ignored. The focus will move in the direction of the cognitive structures of energy - information, the body can be considered as a quantum generator of information: Electromagnetic, solitonic, sound, spin and bioplasma. The term "information" has many meanings, it is generally understood as something that carries a message. Information is also referred to as the ability to organize system or maintain an organized state.

Solitons are independent entities. Soliton is defined as a moving solitary impulse of high-power, which does not blur during the contact with another particle, wave, or the field. There are solitons of light, water and sound, which can strongly interact with other solitons, but after this interaction the form and structure remain unaltered, such as when two soliton waves approach each other they "notice" themselves and penetrate each other, but they do not overlap; then they spread in the same order in which they had been connected. This means that they penetrate each other,

\section{References}

1 Baaquie E, Martin F (2005) Quantum psyche, quantum field theory of the human psyche. Neuro Quantol 3: 7-12.

2 Adamski A (2006) The biological system as an electronic device in the process of learning about the environment and itself: Man-His bioelectronic structure and the perception of music. Pro-Pak Kęty publishing house, Poland.

3 Sedlak W (1976) Materials from the first conference on bioplasms. Catholic University of Lublin, Lublin, Poland.

4 Adamski A (2005) Melanin, enzymes, melatonin in health and disease. Fishpond, Magnum Publisher, Poland.

5 Brizhik L (2000) Energy and information transfer in biological systems: How physics could enrich. Proceedings of the international workshop, Poland.

6 Brizhik L (2003) Soliton mechanism of charge energies and information transfer in biosystem: Energy and information transfer in biological systems. Pro-Pak Kęty publishing house, Poland.

7 Adamski A (2017) Bioplasma as a link between cosmic consciousness and human consciousness and its influence on the creation of artificial consciousness. In: Earth kosmos in a perspective of safety challenges, chances and threats. Pro-Pak Kęty publishing house, Poland.

8 Adamski A (2016) Searching for the nature of consciousness in quantum processes, Publisher of the University of Silesia in Katowice, Katowice, Poland.

9 Heyerhoff $\mathrm{M}$ (1997) The history of the early period of soliton theory. Marienstr, Germany.

10 Scott AC (2005) Encyclopedia of non-linear science, Routledge, Taylor and Francis Group, New York, USA.

11 Russell JS (1844) Report on waves. $14^{\text {th }}$ Meeting of the British association for the advancement of science, York, London, UK.

12 Zabusky NJ, Kruskal MD (1965) Interaction of 'Solitons' in a without losing their identity. Soliton waves carry signals without the necessity of moving the environment, as a carrier wave. Only spatial relations are transmitted that is the geometry of the constellation of particles of water and air without their physical part - the environment contributes to this process only as a structural pattern.

Solition signals are transmitted not only to the biological structures, but also to the psychological and spiritual realm these are our mental, emotional and conscious states. Solitons can spread into the entire universe, and they do not disappear. They have existed from the beginning of life, to the present. The cosmos was densely filled with solitons network, carrying the content and meaning. Information fields (solitons) can affect the energy systems almost without the loss of energy and cause large changes in the biological system. These fields and thoughts can influence each other and are associated not only with the biosphere, but also with the noosphere. Their variety of densities is infinite. The brain has the ability to generate and receive information fields, and therefore, these fields may be a carrier of information from one brain to another.

collisionless plasma and the recurrence of initial states. Phy Rev Lett 15: 240-243.

13 Stegeman G, Segev M (1999) Optical spatial solitons and their interactions: Universality and diversity. Science 286: 15181523.

14 Segev M, Crosignani B, Yariv A, Fischer B (1992) Spatial solitons in photorefractive media. Phy Rev Lett 68: 923-926.

15 Pang X (2000) Vibrational energy-spectra of protein molecules and non-thermally biological effect of infra-red light. Institute of High-Energy Electronics, University of Electronic Science and Technology of China, Chinese Academy of Sciences.

16 Ivanisevic VG, Ivancevic TT (2013) Sine-gordon solitons, kinks and breathers as physical models of nonlinear excitations in living cellular structures. J Geo Symmet Phy 31: 5.

17 Inchauspe A (2016) Therapeutic acupunctural resonance II: New discoveries that justify the outcomes of this new therapeutic modality. J Biosci Med 4: 39-45.

18 Pang XF (2001) Vibrational energy-spectra of protein molecules and non-thermally biological effect of infrared light. Monography for the university of electronic science and technology of chengdu, Chengdu, P.R. China.

19 Pouget J, Maugin GA (1984) Solitons and electroacoustic interactions in ferroelectric crystals: Single solitons and domain walls. Phy Rev 30: 5306.

20 PougeT J, Maugin G (1985) Solitons and electroacoustic interactions in ferroelectric crystals: Interactions of solitons and radiations. Phy Rev B 7: 4633.

21 Matuszewski M (2007) Searching for multidimensional optical solitons using variance methods. Institute of Theoretical Physics at the University of Warsaw, The Trippenbach brand, Poland.

22 Adamski A (2007) The role of bioelectronic processes in shaping sensory perception and human psychological functions. University of Silesia, Katowice, Poland. 
23 Trippenbach M, Infeld E (2007) Non-linear optics of atoms. Adv Phy 58: 55-66.

24 Salasnich L, Parola A, Reatto L (2002) Condensate bright solitons under transverse confinement. Phys Rev A 66: 043603.

25 Chodurek E, Zdybel M, Pilawa B (2013) Application of EPR spectroscopy to examination of free radicals in melanins from A-375 and G-361 human melanoma malignum cells. J Appl Biomed 11: 173-185.

26 Abbas M, Damico F, Morresi L, Pinton A, Ficcadenti M, et al. (2009) Structural, electrical, electronic and optical properties of melanin films. Eur Phys J 28: 285-291.

27 Pilawa B (2003) Effect of oxygen on spin-spin and spin lattice relaxation in DOPA-melanin: Complexes with chloroquine and metal ions. Appl Magn Reason 25: 105-111.

28 Sarna T (1981) Study of the structure and properties of melanin active centers. Zagad Biof Co 6: 201-219.
29 Adamski A (2016) Role of Bose-Einstein condensate and bioplasma in shaping consciousness Neuro Quantol 14: 896907.

30 Popp FA (2003) Food quality analysis using biophotonics. Food Technol 7: 55-66.

31 Akhmediev N, Ankiewicz A (2008) Dissipative solitons: From optics to biology and medicine. Springer, Berlin Heidelberg, New York, USA.

32 Adamski A (2016) The importance of movement, solitons and coherent light in the development of mental processes. J Adv Neurosci Res 3: 24-31.

33 Adamski A (2018) Hawkins attractor fields as determinants of the development of human psychic structures and their interpretation on the ground of quantum psychology. In: Unity and diversity of consciousness in the modeling of the psychic reality. Augustana, Illinois, USA.

34 Trąbka J (2003) Nero-pyychologia of light. Jagiellonian University Krakow, Poland. 